\title{
Determinación de Proporciones Áureas Cráneofaciales para la Reconstrucción con Fines de Identificación Médicolegal
}

\author{
Determination of Golden Craniofacial Proportions for \\ Reconstruction with Medicolegal Identification Purposes
}

"Iván Suazo Galdames; "Erick Gonzalo Trujillo Hernandez; "Mario Cantín López \& *'Daniela Zavando Matamala

\begin{abstract}
SUAZO, G. I.; TRUJILLO, H. E. G.; CANTIN, L. M. \& ZAVANDO, M. D. Determinación de proporciones áureas cráneofaciales para la reconstrucción con fines de identificación médicolegal. Int. J. Morphol., 26(2):331-335, 2008.

RESUMEN: La proporción áurea es una relación matemática que se establece entre dos estructuras, representada numéricamente como 1 es a 1,618. Esta proporción se encuentra presente en la naturaleza y en la arquitectura de la región cráneofacial. En el presente estudio se analizaron las relaciones de proporcionalidad entre rectas formadas por la unión de puntos anatómicos reproducibles y otros antropométricos, identificándose aquellas que se relacionaban de manera áurea. Para ello, se identificaron 20 puntos anatómicos reproducibles medianos y paramedianos, en 10 cráneos humanos con características antropológicas homogéneas. Las rectas obtenidas por la unión de los puntos fueron relacionadas matemáticamente, para identificar las que establecían relaciones áureas. Se encontraron 42 relaciones áuricas entre rectas formadas por la unión de puntos específicos del macizo cráneofacial, que se repiten, o son constantes, en los cráneos estudiados. Se puede aseverar que en la arquitectura de la cabeza ósea se encuentran relaciones proporcionales, cuyo conocimiento puede servir de base para las reconstrucciones con fines de identificación médicolegal.
\end{abstract}

PALABRAS CLAVE: Antropología forense; Identificación humana.

\section{INTRODUCCIÓN}

La proporción áurea es la división armónica de una recta en media y extrema razón, es decir que, el segmento menor, es al segmento mayor, como éste es a la totalidad de la recta. El símbolo de la sección áurea es F (fi), y la representación en números de esta relación de tamaños es 1 es a 1,618. (Rosenthal, 2000). Las proporciones áuricas se encuentran en la naturaleza, con flores de 5, 8 y 13 pétalos, en las circunferencias perfectas de un constel marino, en constelaciones etc. (Seghers et al., 1964).

El hombre presenta en sus dedos una relación áurica de una falange con la siguiente (Jakubietz et al., 2005), además, existen relaciones o proporciones áuricas entre diferentes segmentos óseos del cuerpo humano, de forma tal que es posible establecer una arquitectura ósea basada en proporciones áuricas (Amoric, 1989, 1995; Radiuk, 2001).

Diversos autores han descrito que es posible la reconstrucción de un cráneo a partir de osamentas, utilizando el conocimiento anatómico y las relaciones de proporciona- lidad que establecen los segmentos entre sí (Captier et al., 2003; Nameki et al., 2005).

Desde el punto de vista de la identificación médicolegal, la reconstrucción de la cabeza ósea es fundamental para la aplicación de técnicas como superposición fotográfica y la plastía forense (Gatliff, 1984; Clement \& Ko'sa, 1992; Jayaprakash et al., 2001; Suazo et al., 2007).

El objetivo de este estudio fue determinar la presencia de proporciones áuricas cráneofaciales entre rectas formadas por la unión puntos anatómicos y antropométricos reproducibles.

\section{MATERIAL Y MÉTODO}

Se utilizaron 27 cráneos adultos del Laboratorio de Anatomía Normal de la Universidad de Talca, los cuales se

\footnotetext{
* Unidad de Anatomía Normal, Facultad de Ciencias de la Salud Universidad de Talca, Chile.

** Departamento de Estomatología, Facultad de Ciencias de la Salud, Universidad de Talca, Chile.
} 
encontraban en óptimo estado de conservación y fueron caracterizados antropométricamente con la finalidad de seleccionar una muestra homogénea sobre la cual poder identificar puntos y trazar rectas entre ellos, calculando las proporciones existentes entre rectas.

Caracterización antropométrica: Para identificar antropométricamente los cráneos se partió de la base que todos correspondían a la especie humana. Para la determinación de la tendencia racial se utilizó el método descrito por Rodríguez (2004) que utiliza la forma y dimensiones de las cavidades del cráneo y cara. Para determinar el sexo, se utilizaó un conjunto de factores, como la prominencia y forma de los procesos mastoides, márgenes supraorbitarios, procesos estiloides, protuberancia occipital externa y apoyo horizontal sobre el proceso mastoides (Teke, 2004).

La edad aproximada de los cráneos fue determinada mediante el método de análisis del grado de sinostosis de las suturas craneales (Meindl \&. Lovejoy, 1985).

Para determinar el biotipo se realizó el método propuesto por del Sol (2005), que se resume en la siguiente fórmula: (Diámetro transverso máximo /Diámetro antero posterior máximo) x 100 .

Se seleccionaron 10 cráneos de sujetos adultos, cuya edad promedio era de 49,2 años (DS 10,11), de raza mongoloide, de sexo masculino y biotipo braquicefálico. Sobre estos cráneos se marcaron con un lápiz de tinta indeleble de 0,5 $\mathrm{mm}, 8$ puntos medianos y 12 puntos paramedianos derechos.

\section{Puntos Medianos}

Glabela: punto más prominente de la eminencia roma ubicada entre las crestas supraorbitarias.

Nasion: punto donde la línea mediana intersecta la sutura frontonasal.

Rhinion: punto en la línea mediana, que se corresponde con el borde más anterior de los huesos nasales.

Espina nasal anterior : punto correspondiente a la unión de ambas semiespinas de la cara medial (nasal) del hueso maxilar.

A de Downs: punto más profundo de la concavidad del maxilar.

Foramen nasopalatino: punto más posterior del margen del agujero formado por la unión de los procesos palatinos de ambos maxilares, en el segmento anterior del paladar duro.

Espina nasal posterior: punto formado por la unión de los bordes posteriores del hueso palatino.
Encrucijada palatina: punto formado por la intersección del rafe medio palatino, con la línea que resulta de la división de los procesos palatinos de los huesos maxilares y de los procesos horizontales del hueso palatino.

\section{Puntos Paramedianos}

Proceso frontal lateral: punto más prominente de la eminencia frontal lateral ubicada en la cara anterior del hueso frontal.

Foramen cigomatofacial: agujero simple, ubicado en la cara lateral del hueso cigomático.

Incisura supraorbitaria: punto más profundo del margen de la incisura supraorbitaria del hueso frontal.

Proceso mastoides: vértice del proceso mastoides del hueso temporal.

Foramen infraorbitario: punto ubicado en la parte más profunda del margen superior del agujero infraorbitario.

Sutura frontocigomática: corresponde al punto medio de la unión de los huesos frontal y cigomático.

Fosa mandibular: corresponde al punto más profundo de la concavidad ubicada en la porción escamosa de la cara inferior del hueso temporal.

Tubérculo cigomático anterior: corresponde al punto más prominente de la eminencia ubicada en la cara inferior del proceso cigomático del hueso temporal.

Foramen redondo: punto más lateral del agujero redondo, ubicado en el borde medial de las alas mayores del esfenoides.

Sutura cigomatotemporal: punto medio de la unión de los huesos cigomático y temporal.

Foramen palatino mayor: punto más posterior del margen del agujero palatino mayor del hueso maxilar.

Espina del esfenoides: punto más prominente de la espina que se forma por la unión de los bordes lateral y medial del ala mayor del esfenoides.

Una vez marcados los puntos, se procedió a medir la distancia existente entre ellos, utilizando un caliper manual $(0,1 \mathrm{~mm})$, determinando rectas, las que se denominaron de acuerdo a los puntos entre los cuales se encontraban. Se obtuvieron un total de 190 rectas por cada cráneo, las cuales se compararon todas versus todas, observando la relación matemática existente entre ellas, para así seleccionar aquellas que se encontraban en una proporción áurica, es decir 1 es a 1,618. 


\section{RESULTADOS}

Tabla I. Proporciones áuricas cráneofaciales presentes en cráneos adultos del Laboratorio de Anatomía de la Universidad de Talca, p = 0,05.

\section{0}

Rhinion - Espina nasal anterior

Nasion - Foramen cigomatofacial

Espina nasal posterior - Sutura cigomatotemporal

Foramen cigomatofacial - Espina del esfenoides

Nasion - Foramen cigomatofacial

Foramen nasopalatino - Espina nasal posterior

Nasion - Sutura cigomatotemporal

Rhinion - Sutura cigomatotemporal

9 Foramen nasopalatino - Espina del esfenoides

10 Nasion - Foramen cigomatofacial

11 Espina nasal posterior - Fosa mandibular

12 Espina nasal posterior - Sutura cigomatotemporal

13 Foramen infraorbitario - Tubérculo cigomático anter ior

14 Espina nasal anterior - Sutura frontocigomática

15 Encrucijada palatina - Sutura frontocigomática

16 Encrucijada palatina - Espina del esfenoides

17 Espina nasal posterior - Foramen infraorbitario

18 Espina nasal anterior - Foramen supraorbitario

19 Nasion - Espina nasal posterior

20 Encrucijada palatina - Tubérculo cigomático anterior

21 Glabela - Sutura frontocigomática

22 Nasion - Sutura frontocigomática

23 Glabela - Foramen infraorbitario

24 Encrucijada palatina - Foramen redondo

Foramen infraorbitario - Fosa mandibular

Espina nasal posterior - Foramen infraorbitario

Encrucijada palatina - Foramen redondo

Espina nasal posterior - Foramen infraorbitario

Encrucijada palatina - Sutura cigomatotemporal

Foramen infraorbitario - Fosa mandibular

Espina nasal posterior - Fosa mandibular

Espina nasal posterior - Sutura cigomatotemporal

Encrucijada palatina - Sutura cigomatotemporal

Nasion - Encrucijada palatina

Espina nasal posterior - Sutura cigomatotemporal

Encrucijada palatina - Sutura cigomatotemporal

Foramen infraorbitario - Tubérculo cigomático anterior

Nasion - Foramen cigomatofacial

Espina nasal posterior - Sutura cigomatotemporal

Encrucijada palatina - Sutura cigomatotemporal

Rhinion - Foramen palatino may or

Foramen infraorbitario - Foramen redondo

\subsection{8}

Glabela - Espina nasal anterior.

Glabela - Espina del esfenoides

Glabela - Espina del esfenoides

Glabela - Espina del esfenoides

Glabela - Tubérculo cigomático anterior

Nasion - Espina nasal posterior

Rhinion - Proceso mastoideo

Rhinion - Proceso mastoideo

Rhinion - Proceso mastoideo

Espina nasal anterior - Proceso frontal lateral

Espina nasal anterior - Proceso frontal lateral

Espina nasal anterior - Proceso frontal lateral

Espina nasal anterior - Proceso frontal lateral

Espina nasal anterior - Proceso mastoideo

Espina nasal anterior - Proceso mastoideo

Espina nasal anterior - Espina del esfenoides

Espina nasal anterior - Sutura frontocigomática

A de Downs - Proceso frontal lateral

Foramen nasopalatino - Proceso mastoideo

Foramen nasopalatino - Proceso mastoideo

Foramen nasopalatino - Fosa mandibular

Foramen nasopalatino - Fosa mandibular

Foramen nasopalatino - Tubérculo cigomático anterior

Foramen nasopalatino - Tubérculo cigomático anterior

Encrucijada palatina - Proceso frontal lateral

Encrucijada palatina - Foramen supraorbitario

Encrucijada palatina - Proceso mastoideo

Encrucijada palatina - Sutura frontocigomática

Proceso frontal lateral - Espina del esfenoides

Proceso frontal lateral - Espina del esfenoides

Proceso frontal lateral - Fosa mandibular

Proceso frontal lateral - Fosa mandibular

Proceso frontal lateral - Fosa mandibular

Proceso frontal lateral - Tubérculo cigomático anterior

Proceso frontal lateral - Tubérculo cigomático anterior.

Proceso frontal lateral - Tubérculo cigomático anterior

Proceso frontal lateral - Tubérculo cigomático anterior

Proceso frontal lateral - Foramen redondo

Proceso frontal lateral - Foramen palatino mayor

Proceso frontal lateral - Foramen palatino mayor

Proceso mastoideo - Sutura frontocigomática

Proceso mastoideo - Sutura frontocigomática 
La muestra estuvo integrada por 10 cráneos de características antropométricas homogéneas. En cada cráneo se identificaron y marcaron 20 puntos, los cuales al unirse en pares determinaron la formación de 190 rectas por cada cráneo.

Se realizaron las confrontaciones de las rectas de cada cráneo, dando como resultado 36.100 proporciones por cada cráneo, lo que hace un total de 361.000 proporciones en el total de la muestra, de las cuales, 42 resultaron ser proporciones áuricas presentes en todas las muestras $(\mathrm{p}=0,05)$.

En la Tabla I, se observan las relaciones entre rectas formadas por la unión de puntos que se encontraron en proporción áurea.

\section{DISCUSIÓN}

La proporción áurea, corresponde a una relación en la cual una parte se relaciona con la otra como 1 es a 1,618, ésta relación la encontramos distintos niveles de organización en la naturaleza. En la arquitectura del cuerpo huma- no también se encuentra presente este principio de proporcionalidad (Jakubietz et al.).

En el presente estudio se analizaron las relaciones de proporcionalidad entre rectas trazadas por la unión de puntos anatómicos reproducibles y otros antropométricos, identificándose aquellas que se relacionaban de manera áurea. Los resultados permiten identificar 42 relaciones áureas constantes en todos los cráneos de la muestra, por lo que pueden considerarse como proporciones estables que nos servirían como punto de partida para la reconstrucción craneofacial con fines de identificación médicolegal a partir de cráneos incompletos u osamentas, ya que de acuerdo con lo referido por algunos autores la reconstrucción de la cabeza ósea es necesaria antes de realizar técnicas como la plastía forense (Ubelaker \& O'Donnell, 1992; Suazo et al.) o la superposición foto-radiográfica craneofacial (AustinSmith \& Maples, 1994; Yoshino et al., 1995) y que ésta puede realizarse utilizando las relaciones proporcionales que los distintos puntos de la cabeza establecen.

Se puede aseverar que en la arquitectura de la cabeza ósea se encuentran relaciones proporcionales cuyo conocimiento puede servir de base para las reconstrucciones con fines de identificación médicolegal.

SUAZO, G. I.; TRUJILLO, H. E. G.; CANTIN, L. M. \& ZAVANDO, M. D. Determination of golden craniofacial proportions for reconstruction with medicolegal identification purposes.Int. J. Morphol., 26(2):331-335, 2008.

SUMMARY: The golden proportion is a mathematical relation that establishes between two structures represented numerically like 1 is to 1.618. This proportion is present in the nature and in the craniofacial architecture. In the present study analyzed the proportionality relations of anatomical and other anthropometric points between straight lines planned by the union of anatomical points, identifying those that were relating in a golden way, for it 20 medium and paramedium points was identificated in 10 human crania, the lines obtained by the union of the points were related mathematically, they identified those who were establishing golden relations. They found 42 golden relations between straight lines formed by the union of specific points of the craniofacial region, which repeat themselves, or they are constant in the studied crania. It is possible to affirm that in the architecture of the bony head they find proportional relations which knowledge can use as base for the reconstructions with ends of medicolegal identification.

KEY WORDS: Forensic anthropology; Human identification.

\section{REFERENCIAS BIBLIOGRÁFICAS}

Amoric, M. The golden number. Applications to architectural and structural cranio-facial analysis, Actual Odontostomatol. (Paris), 42(166):205-19, 1989.

Amoric, M. The golden number: Applications to cranio-facial evaluation. Funct. Orthod., 12(1):18-21, 24-5, 1995.

Austin-Smith, D. \& Maples, W. The reliability of skull/ photograph superimposition in individual identification. J. Forensic Sci., 39(2):446-55, 1994.
Captier, G.; Leboucq, N.; Bigorre, M.; Canovas, F.; Bonnel, F.; Bonnafe, A. \& Montoya, P. Plagiocephaly: morphometry of skull base asymmetry. Surg. Radiol. Anat., 25(2-3):226-33, 2003.

Clement, J. \& Ko'sa, F. The fetal skeleton. In: Clark, D. H. (Ed.). Practical Forensic Odontology. Oxford, Ed. Wright, 1992. pp.43-52.

del Sol, M. Índice cefálico en un grupo de individuos 
mapuches de la IX Región de Chile. Int. J. Morphol., 23(3):241-6, 2005.

Gatliff, B. Facial sculpture on the skull for identification. Am. J. Med. Pathol., 5(4):327-32, 1984.

Jakubietz, R.; Jakubietz, M.; Kloss, D. \& Gruenert, J. Defining the Basic Aesthetics of the Hand. Aesth. Plast. Surg., 29(6):546-51, 2005.

Jayaprakash, P.; Srinivasanb, G. \& Amravaneswaran, M. Cranio-facial morphanalysis: a new method for enhancing reliability while identifying skulls by photo superimposition. Forensic Sci. Int.,117(1-2):121-43, 2001.

Meindl, R. \& Lovejoy, C. Ectocranial suture closure: A revised method for the determination of skeletal age at death based on the lateral-anterior sutures. Am. J. Phys. Anthrop,.68(1):57-66, 1985.

Nameki, H.; Kato, T.; Nameki, I. \& Ajimi, Y. Selective reconstructive options for the anterior skull base. Int. J. Clin. Oncol., 10(4):223-8, 2005.

Radiuk, M. The biological essence of golden sections. $Z h$. Obshch. Biol., 62(5):403-9, 2001.

Rodríguez, J. V. La Antropología Forense en la identificación humana. Bogotá, Ed. Anaconda, 2004. p259.

Rosenthal, E. A. Of golden proportions. J. Hand Ther., 13(1):6-11, 2000.

Seghers, M. J., Longacre, J. J. \& deStefano, G. A. The golden proportion and beauty. Plast. Reconstr. Surg., 34:382, 1964.
Suazo, G. I. C.; Pérez, R. F. J. \& Torres, M. S. R. Grosores titulares faciales en cadáveres de españoles y su aplicación en la identificación médicolegal. Int. J. Morphol., 25(1):109-16, 2007.

Teke, A. Medicina Legal y Criminalística. Santiago de Chile, Editorial Jurídica de Santiago, 2004.

Ubelaker, D. H. \& O'Donnell, G. Computer-assisted facial reproduction. J. Forensic Sci., 37(1):155-62, 1992.

Yoshino, M.; Imaizumi, K.; Miyasaka, S. \& Seta, S. Evaluation of anatomical consistency in cranio-facial superimposition images. Forensic Sci. Int., 74(1-2):12534, 1995.

Dirección para correspondencia:

Prof. Iván Claudio Suazo Galdames

Oficina 104 Anatomía Normal,

Universidad de Talca

Campus Lircay.

Avda Lircay S/N, Talca VII Región

CHILE

Teléfono: $56-71-201682$

Fax: 56-71-200452

E mail: isuazo@utalca.cl

Recibido : 02-08-2007

Aceptado: 06-03-2008 
